\title{
Incorporated glucosamine adversely affects the emulsifying properties of whey protein isolate polymerized by transglutaminase
}

\author{
Lin Chen, ${ }^{* 1}$ Niamat Ullah, ${ }^{* 1}$ Chenyi Li, ${ }^{*}$ Robert M. Hackman, $†$ Zhixi Li, ${ }^{*}$ Xinglian Xu, $\ddagger$ Guanghong Zhou, $\ddagger$ \\ and Xianchao Feng*2 \\ ${ }^{*}$ College of Food Science and Engineering, Northwest A\&F University, No. 22 Xinong Road, Yangling, Shaanxi 712100, China \\ †College of Agricultural and Environmental Sciences, University of California, Davis, 1 Shields Avenue, Davis 95616 \\ †Laboratory of Meat Processing and Quality Control of Ministry of Education, College of Food Science and Technology, \\ Synergetic Innovation Center of Food Safety and Nutrition, Nanjing Agricultural University, Nanjing, Jiangsu 210095, China
}

\section{ABSTRACT}

Glucosamine $(\mathrm{GlcN})$ and microbial transglutaminase (Tgase) are used separately or together to improve the emulsifying properties of whey protein isolate (WPI). However, little is known about how the emulsifying properties change when GlcN residues are incorporated into WPI cross-linked by Tgase. We used Tgase as a biocatalyst to cross-link WPI in the presence of GlcN in a liquid system for $12 \mathrm{~h}$ at a moderate temperature $\left(25^{\circ} \mathrm{C}\right)$. Matrix-assisted laser desorption/ionization time-of-flight mass spectrometry analyses indicated that protein polymerization and GlcN conjugation occurred simultaneously, phenomena also supported by the loss of free amines (9.4-20.5\%). Addition of 5 $\mathrm{U}$ Tgase/g protein improved the emulsifying properties of moderately cross-linked WPI polymers. The Tgase-treated WPI polymers had a larger particle size ( 2.6 -fold) than native WPI, which may have reduced coalescence and flocculation in an oil-in-water emulsion system. However, the incorporation of GlcN residues into WPI, predominantly via enzymatic glycation, partly inhibited the cross-links between the WPI molecules catalyzed by Tgase, reducing the size of the WPI polymers 0.81 - to 0.86 -fold). Consequently, WPI+GlcN conjugates provided less stability to the emulsion. Moreover, high $\mathrm{NaCl}$ concentration $(0.2 M)$ decreased the emulsifying properties of the WPI+GlcN conjugates by neutralizing negative electric charges in the glycoconjugates. However, the improved emulsifying properties of WPI cross-linked by Tgase may be useful in food processing at higher $\mathrm{NaCl}$ concentrations due to the formation of the thicker steric barrier at the oil-water interface.

Received September 29, 2016.

Accepted January 22, 2017.

${ }^{1}$ These authors contributed equally to this study.

${ }^{2}$ Corresponding author: fengxianchao1@hotmail.com
Key words: cross-linked whey protein isolate, glucosamine, microbial transglutaminase, whey protein isolate and glucosamine conjugates

\section{INTRODUCTION}

Whey protein isolate (WPI) is widely used in food processing to promote emulsification, stabilization, foaming, and gelation in a variety of foodstuffs (Sarbon et al., 2015). Although native WPI have many beneficial properties, researchers have attempted to develop new WPI-based ingredients with wider applications by enhancing the functionalities of WPI with physicochemical or enzymatic treatments.

As a naturally occurring reaction, glycation can improve the functional properties of proteins and even impart novel functionality. Glycation can improve the foaming properties of $\beta$-lactoglobulin and bovine sodium caseinate, broadening their applications as foaming agents, mainly in acidic foods. The emulsifying properties and thermal stability of some other proteins have also been upgraded via the Maillard reaction (Hrynets et al., 2013).

Maillard-type glycation is highly accelerated by heat treatment and is usually employed to modify proteins in a dry state (Hrynets et al., 2013). However, the drystate process has limited the application of glycation in the food industry. Furthermore, the dry-state reaction is difficult to control and has a long reaction time (Hrynets et al., 2013). Glycation in a liquid state could overcome these problems and would be more viable for the food industry. Furthermore, methods that add enzymatic treatments have been proposed to increase glycoconjugation at a lower temperature in a liquid state and limit the formation of advanced glycation end products (Hrynets et al., 2013). Of the potential glycation enzymes, transglutaminase (Tgase) is widely available, high yielding, and inexpensive (Hrynets et al., 2013). In general, Tgase can catalyze 2 typical reactions: (A) protein cross-linking and (B) incorporation of 

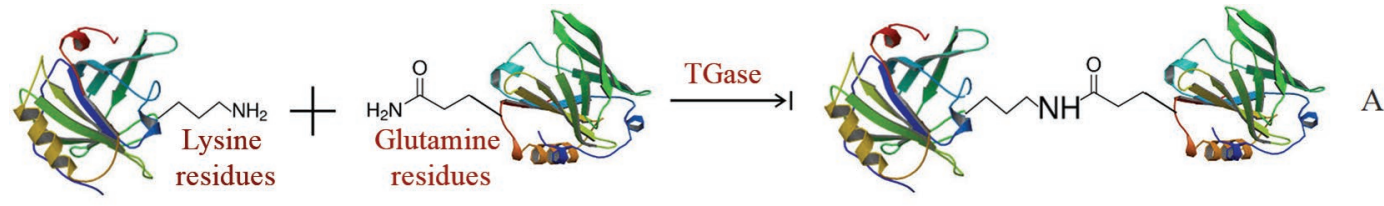

A
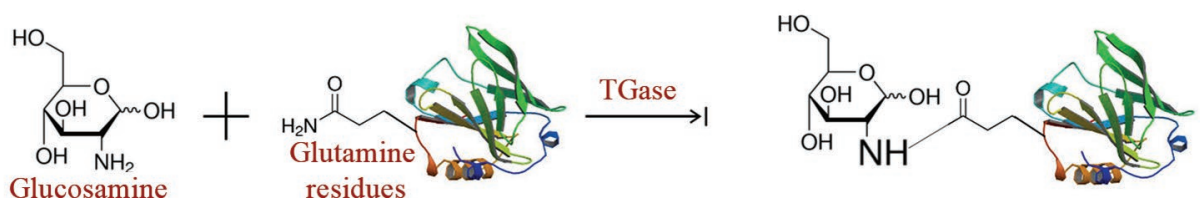

B

Figure 1. Two typical reactions catalyzed by transglutaminase (Tgase). Color version available online.

aminosaccharides into proteins through an acyl transfer reaction in protein substrates (Figure 1).

Glucosamine $(\mathbf{G l c N})$ is a naturally occurring aminosaccharide that has higher reactivity in the Maillard reaction than glucose and can be incorporated into proteins by Tgase (Arbia et al., 2013; Hrynets et al., 2014). Because GlcN possesses both amino and hydrophilic hydroxyl groups, it is a promising compound for improving the functionality of proteins (Jiang and Zhao, 2010; Hrynets et al., 2013,2014). The emulsifying activity of proteins is increased when they are treated with Tgase (Song and Zhao, 2014), but other studies have shown that using Tgase to improve cross-linking and incorporate GlcN residues into protein also improves emulsifying properties (Hrynets et al., 2014). However, the influence of GlcN on the protein cross-links induced by Tgase, and thus on the emulsifying properties of the protein, has been scarcely documented.

We planned this study to elucidate the influence of GlcN on the emulsifying properties of WPI treated with Tgase. In this work, WPI were to be treated with Tgase and GlcN. We analyzed the physicochemical properties of the treated WPI using matrix-assisted laser desorption/ionization time-of-flight (MALDI-TOF) mass spectrometry and dynamic light scattering (DLS). We also assessed differences in the performance of WPI as emulsifiers after different treatments in emulsifying activity and stability. We analyzed the microstructure of oil-in-water emulsions prepared with treated WPI using confocal laser scanning microscopy. Understanding the fundamental reactions between Tgase and WPI in the presence of GlcN will enable us to optimize the formula used to improve the qualities of food products.

\section{MATERIALS AND METHODS}

\section{WPI Treated with GIcN and Tgase}

Whey protein isolate (5\%) was mixed with $\mathrm{GlcN}$ in ratios of 1:0, 1:1, and 1:3 (wt/wt) in $20 \mathrm{~mL}$ of deionized water. Depending on the addition of Tgase $(0$ and $5 \mathrm{U}$ of Tgase/g of WPI), we prepared 6 different reaction mixtures in triplicate, according to a total factorial design. All samples were incubated using a Hy-4 shaker (Zhiboru Equipment Manufacture Co., Ltd., Changzhou, Jiangsu, China) under constant agitation for $12 \mathrm{~h}$ at $25^{\circ} \mathrm{C}$. All samples were then heated at $85^{\circ} \mathrm{C}$ for $5 \mathrm{~min}$, inactivating the Tgase in the samples that contained it. After the samples were cooled with running tap water for $1 \mathrm{~h}$, they were dialyzed against deionized water at $4^{\circ} \mathrm{C}$ for $16 \mathrm{~h}$, with 4 changes of water to remove free GlcN. Samples that contained neither GlcN nor Tgase served as controls.

\section{Free Amines Analysis}

Free amine content in proteins was analyzed using methods described previously (Feng et al., 2015b). Briefly, WPI samples were diluted with SDS solution (1\%) to $0.2 \mathrm{mg} / \mathrm{mL}$ in $0.1 \mathrm{M} \mathrm{Na}_{2} \mathrm{~B}_{4} \mathrm{O}_{7}$ (pH 9.3). One milliliter of the $0.2 \mathrm{mg} / \mathrm{mL}$ sample was then mixed with $50 \mu \mathrm{L}$ of $0.03 M$ 2,4,6-trinitrobenzenesulfonic acid (TNBS) and incubated for $1 \mathrm{~h}$ at $37^{\circ} \mathrm{C}$. The whole process was protected from light. Absorbance was measured at $420 \mathrm{~nm}$ after reaction with TNBS. Free amine content was expressed as micromoles of free amines per milligram of protein using a standard curve constructed from glycine.

\section{Evaluation of Glycoconjugation by MALDI-TOF-MS}

The WPI samples treated with GlcN and Tgase were diluted to $0.5 \mathrm{mg} / \mathrm{mL}$ with $50 \mathrm{~m} M \mathrm{NH}_{4} \mathrm{HCO}_{3}$. The, the WPI samples $(3 \mathrm{~mL})$ were subjected to overnight proteolytic digestion at $37^{\circ} \mathrm{C}$ with $150 \mu \mathrm{L}$ of trypsin $\left(0.5 \mathrm{mg} / \mathrm{mL}\right.$ in $\left.50 \mathrm{~m} M \mathrm{NH}_{4} \mathrm{HCO}_{3}\right)$. The hydrolysate was filtered through a $0.45-\mu \mathrm{m}$ filter $(3 \mathrm{kDa}$; Amicon Ultra; Millipore Corporation, Bedford, MA) by centrifugation $(3,000 \times g, 20 \mathrm{~min})$. Then, the filtrate at the bottom of the tube was collected and lyophilized. 
Samples were redissolved with $50 \%$ acetonitrile containing $0.1 \%$ trifluoroacetic acid. The sample solution $(1 \mu \mathrm{L})$ was mixed with $1 \mu \mathrm{L}$ of matrix solution $(10 \mathrm{mg} /$ $\mathrm{mL} \alpha$-cyano-4-hydroxy-cinnamic acid prepared in $50 \%$ acetonitrile containing $0.1 \%$ trifluoroacetic acid) for analysis. All mass spectra were obtained using MALDITOF performed on an Axima Performance instrument (Shimadzu, Tokyo, Japan).

\section{Measurement of WPI Particle Diameter}

We measured the average particle diameter of the protein aggregates by DLS using the Zetasizer Nano ZS (Malvern Instruments Ltd., Malvern, UK). Samples were diluted with deionized water to a final concentration of $1 \mathrm{mg} / \mathrm{mL}$. Cuvettes were filled with $1.1 \mathrm{~mL}$ of sample. For each sample, we conducted 3 measurements with at least 5 runs; each run lasted for $10 \mathrm{~s}$. The aggregates in the sample were expressed as the intensity measured by Nano ZS.

\section{Total Sulfhydryl and Disulfide Bond Content}

The sulfhydryl content of the WPI samples was measured using 5,5'-dithiobis (2-nitrobenzoic acid) (DTNB). The disulfide bond content in WPI samples was measured using disodium 2-nitro-5-thiosulfobenzoate (NTSB). Absorbance at $412 \mathrm{~nm}$ was measured using a spectrophotometer. Absorption coefficients of $11,400 \mathrm{M}^{-1} \mathrm{~cm}^{-1}$ and 13,600 $\mathrm{M}^{-1} \mathrm{~cm}^{-1}$ were used for analysis of free sulfhydryls and disulfide bonds, respectively (Feng et al., 2015a).

\section{Surface Hydrophobicity Analysis}

We analyzed surface hydrophobicity using 8-anilino1-naphthalenesulfonic acid (ANS) as a fluorescence indicator. Samples were centrifuged $(10,000 \times g, 10$ min at $\left.4^{\circ} \mathrm{C}\right)$. We prepared a concentration gradient of WPI solutions $(0.2-5 \mathrm{mg} / \mathrm{mL})$ in $0.1 M$ PBS buffer $(\mathrm{pH}$ 7.0). For each concentration, $3 \mathrm{~mL}$ was mixed with 10 $\mu \mathrm{L}$ of $15 \mathrm{~m} M$ ANS by vortex. After $5 \mathrm{~min}$ at room temperature, the fluorescence intensity was recorded using a F-4600 fluorescence spectrophotometer (Hitachi, Tokyo, Japan) with excitation at $390 \mathrm{~nm}$ and emission at $470 \mathrm{~nm}$. An index of the surface hydrophobicity was indicated by the initial slope of the linear regression of fluorescence intensity against protein concentration.

\section{Emulsifying Activity Index and Emulsion Stability Index Assays}

Samples of WPI solution $(2.5 \%)$ were prepared for 2 treatment groups. Sodium chloride was added into the
WPI solutions to obtain final concentrations of either $20 \mathrm{~m} M$ (low group) or $200 \mathrm{~m} M$ (high group) $\mathrm{NaCl}$. The $\mathrm{pH}$ of the solutions was adjusted to $\mathrm{pH} 7$ with 1 $M \mathrm{HCl}$ or $1 M \mathrm{NaOH}$. Soybean oil (10\% wt/wt) was added into the WPI solutions, which were then emulsified using a high-speed homogenizer (IKA T18-Digital, Staufen, Germany). After homogenization (10,000 rpm for $1 \mathrm{~min}$ ), $50 \mu \mathrm{L}$ of the emulsion was taken from the bottom of the test tube at 0 and $10 \mathrm{~min}$ and diluted it into $5 \mathrm{~mL}$ of $0.1 \%$ SDS. Absorbance (at $500 \mathrm{~nm}$ ) of the suspension was recorded using a $1 \mathrm{~cm}$ path cuvette at $25^{\circ} \mathrm{C}$ (Hrynets et al., 2013). We calculated the emulsifying activity index (EAI) and emulsion stability index (ESI) using the following equations:

$$
\begin{gathered}
\mathrm{EAI}=2.33 \times \mathrm{A}_{0}, \\
\mathrm{ESI}=10 \times\left[\mathrm{A}_{0} /\left(\mathrm{A}_{0}-\mathrm{A}_{10}\right)\right],
\end{gathered}
$$

where $A_{0}$ and $A_{10}$ were the absorbance determined at 0 and $10 \mathrm{~min}$, respectively.

\section{Confocal Laser Scanning Microscopy}

The microstructure of the oil-in-water emulsions containing WPI-GlcN conjugates were imaged with a confocal laser scanning microscope (CLSM; A1R; Nikon Inc., Tokyo, Japan). Fast Green and Nile Red were used as fluorescent dyes for the protein and oil phases, respectively. Each freshly prepared emulsion sample $(100 \mu \mathrm{L})$ was stained with about $10 \mu \mathrm{L}$ of a $0.038 \%$ Fast Green (wt/vol) and $0.038 \%$ Nile Red (wt/ vol) dye mixture for $15 \mathrm{~min}$. The CLSM images were examined with a $10 \times$ magnification lens. A proteincoated oil droplet would be predominantly yellow in the overlay image (green + red $=$ yellow $)$.

\section{Statistical Analyses}

Data (for all samples in triplicate) were collected and subjected to statistical analyses. We applied ANOVA (SPSS 20.0, Chicago, IL) to assess the effect of different treatments. We applied a Tukey's post hoc test when ANOVA found significant differences between treatments. Statistical significance was set at $P<0.05$.

\section{RESULTS AND DISCUSSION}

\section{WPI Conjugation}

In WPI and GlcN mixtures treated with or without Tgase, the free amine levels decreased in a GlcN dosedependent manner (Figure 1). This indicated that GlcN addition enhanced Maillard-type glycation, resulting in 
a decrease of free amine levels in WPI. Hrynets et al. (2013) also found a higher degree of glycation in actomyosin treated with a higher ratio of GlcN. The WPI treated with Tgase (WPI+Tgase) had significantly lower free amine levels at any GlcN dose than WPI treated without Tgase (WPI-Tgase; Figure 2). Transglutaminase conjugates the primary amines of lysine residues (i.e., acyl acceptor) to the glutamine residues (i.e., acyl donor) of the proteins to form intra- and intermolecular $\varepsilon$-( $\gamma$-glutamyl) lysine cross-links (Song and Zhao, 2014). This may contribute to a reduction in the free amines in WPI samples treated with Tgase.

We used MALDI-TOF mass spectrometry to analyze the nature of the covalent bonds between the GlcN residues and WPI. A mass shift of +162 Da between the control (peptides from WPI-Tgase-GlcN) and glycated peptides (peptides from WPI+Tgase) was attributable to attachment of GlcN as a result of enzymatic glycation (Tgase), with the loss of ammonia $\left(\mathrm{NH}_{3}\right)$. On the other hand, a difference of $+161 \mathrm{Da}$ indicated a dehydration-condensation reaction between GlcN and WPI as a result of non-enzymatic glycation (Maillard reaction), with the loss of a water molecule $\left(\mathrm{H}_{2} \mathrm{O}\right)$. Moreover, as shown by Hrynets et al. (2014), a mass shift of +161 Da represents the Schiff base formation between a GlcN residue and WPI at the initial stages of the Maillard reaction, and a +144 Da shift in molecular mass indicates glycation due to the loss of both water and ammonia. Consequently, the presence of both the +161 Da and +144 Da shifts would indicate the occurrence of non-enzymatic glycation. The mass spectrometry results showed that peptides from WPI mixed with GlcN only (-Tgase) were glycated though non-enzymatic glycation (Figure 3 ). The relative number of glycated peptides rose with increasing GlcN dose (Figure 3A, B, and C). Peptides from WPI+Tgase had greater mass than controls, indicating that some proteins were cross-linked by Tgase (Figure 3D). The relative signal intensities of these larger peptides decreased with the addition of GlcN, and the $+162 \mathrm{Da},+161$ $\mathrm{Da}$, and $+144 \mathrm{Da}$ shifts began to appear (Figure 3E and $\mathrm{F}$ ). These results indicated that $\mathrm{GlcN}$ residues can be incorporated into protein through both enzymatic (Tgase) and non-enzymatic glycation (Maillard reaction), both of which inhibited the cross-links between WPI peptides otherwise catalyzed by Tgase. A higher incidence of +162 Da shifts indicated that attachment of GlcN residues to the protein via Tgase dominated and prevented the occurrence of cross-links between WPI catalyzed by Tgase. These results were in accordance with those of Hrynets et al. (2014).

Analysis of DLS can be used to indirectly evaluate changes in the molecular weight of the conjugates

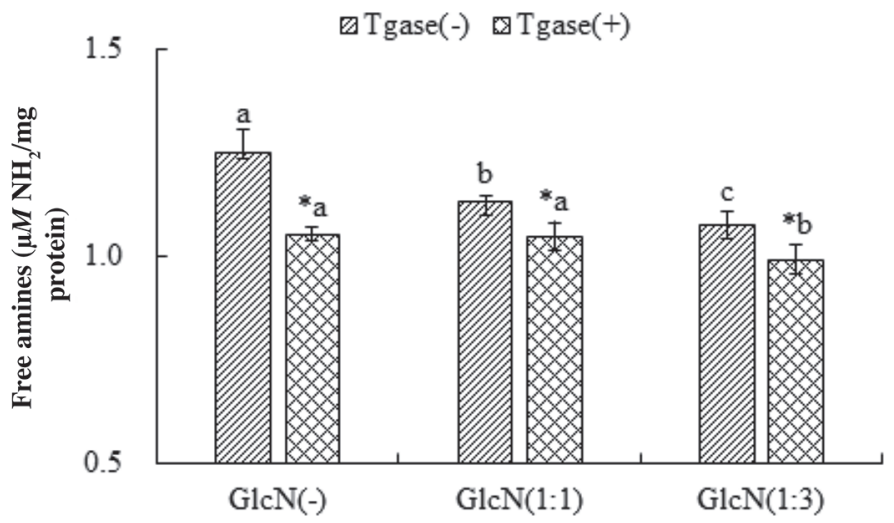

Figure 2. Free amines content in whey protein isolate (WPI) conjugates with different additives. Ratios reflect amount of glucoasamine (GlcN):WPI. *Significant compared with WPI treated without transglutaminase (Tgase). Different letters $(\mathrm{a}-\mathrm{c})$ for WPI \pm Tgase indicate a significant difference $(P<0.05)$. Error bars indicate \pm SD.

treated with GlcN (WPI+GlcN; Figure 4). The average diameter of the WPI+GlcN conjugates increased with the increase in GlcN dose (Figure 4). These changes in the average size of the WPI+GlcN conjugates indicated that part of the WPI treated with GlcN might be glycated. Hrynets et al. (2013) also found that natural actomyosin can be glycated by GlcN in a liquid system at moderate temperatures without Tgase. The average diameters of the WPI conjugated with Tgase were larger than the WPI generated without Tgase (Figure 4), indicating that the addition of Tgase may promote polymerization of WPI. This polymerization may also be seen in the higher band intensities at the top of the separating gels in the WPI+Tgase samples (lanes 2, $4,6)$ compared with the WPI-Tgase (Supplementa1 Figure S1; http://dx.doi.org/10.3168/jds.2016-12071). Also noteworthy is the decrease in the average diameter of the WPI samples treated with Tgase as GlcN increased (Figure 4). This decrease may have been due to a slight inhibition of protein cross-linking (Figure 1A) from the incorporation of small primary amines (GlcN) by Tgase into the protein (Figure 1B). The correlation of this decrease with Tgase-catalyzed incorporation of GlcN is further supported by the decreased band intensities in the WPI+Tgase samples seen at the top of the separating gels (Supplemental Figure S1; https://doi.org/10.3168/jds.2016-12071). Moreover, the band patterns of the WPI samples treated without $\beta$-mercaptoethanol were not different from those treated with $\beta$-ME (Supplemental Figures S1A and B), indicating that the cross-linking in the WPI and WPI+GlcN conjugates was not caused by disulfide bonds.

To further discern the nature of the cross-linking in the WPI conjugates, we determined the content of 
sulfhydryls and disulfide bonds. We observed no significant differences in sulfhydryl or disulfide bond content between any of the WPI samples (Figure 5), indicating that the influence of SH/S-S (sulfhydryl/disulfide) interchange on the emulsifying properties of WPI treated with GlcN and Tgase might be negligible. A previous study found that $\mathrm{SH} / \mathrm{S}-\mathrm{S}$ interchange could reduce the aggregation of oil droplets (Zhu et al., 2010).
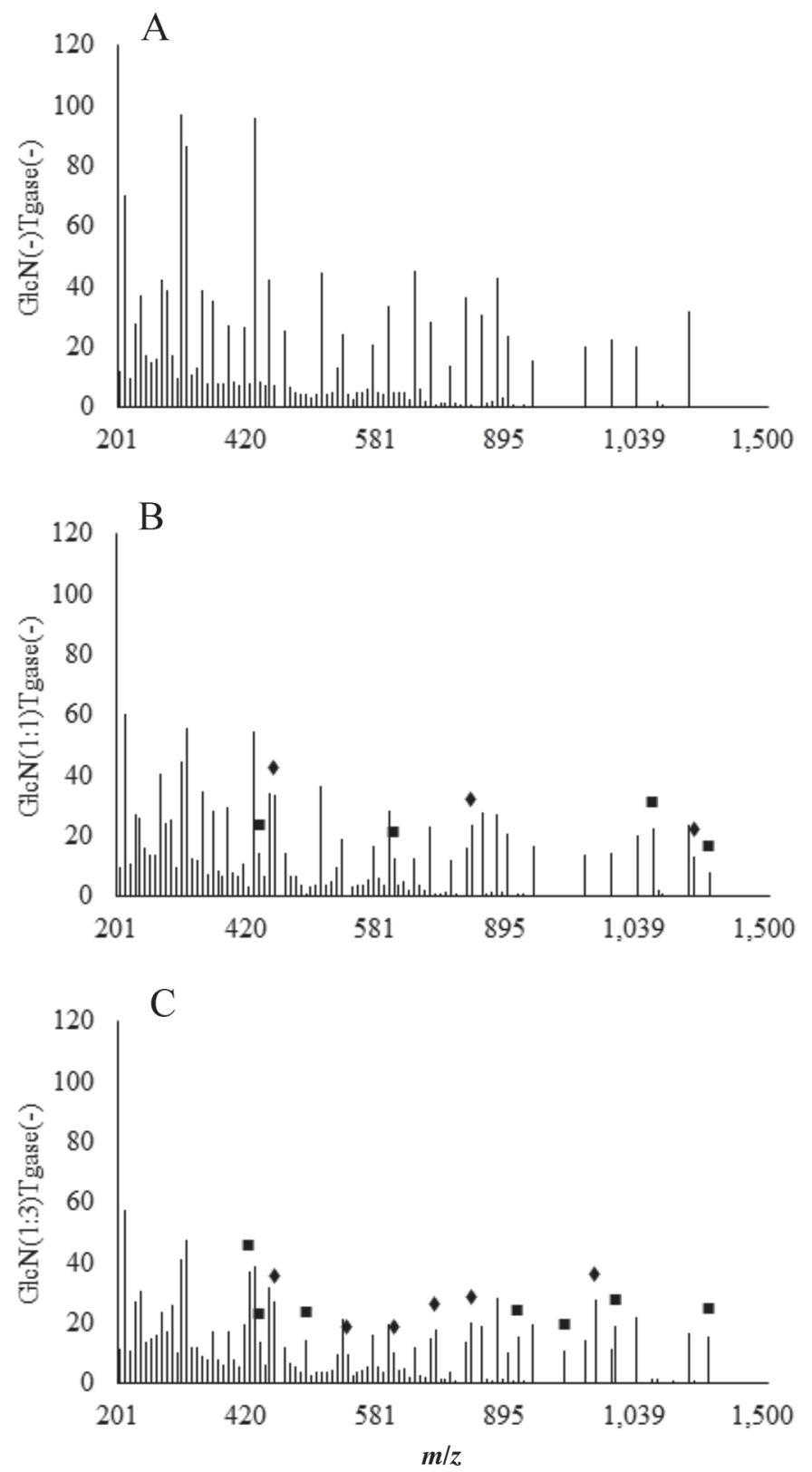

\section{Surface Hydrophobicity}

Surface hydrophobicity is a marker of protein unfolding. Regardless of treatment with Tgase, the surface hydrophobicity significantly decreased in a GlcN dosedependent manner compared with the original WPI (Figure 6). Glycation may change the amphiphilic properties of WPI, making it reasonable to propose
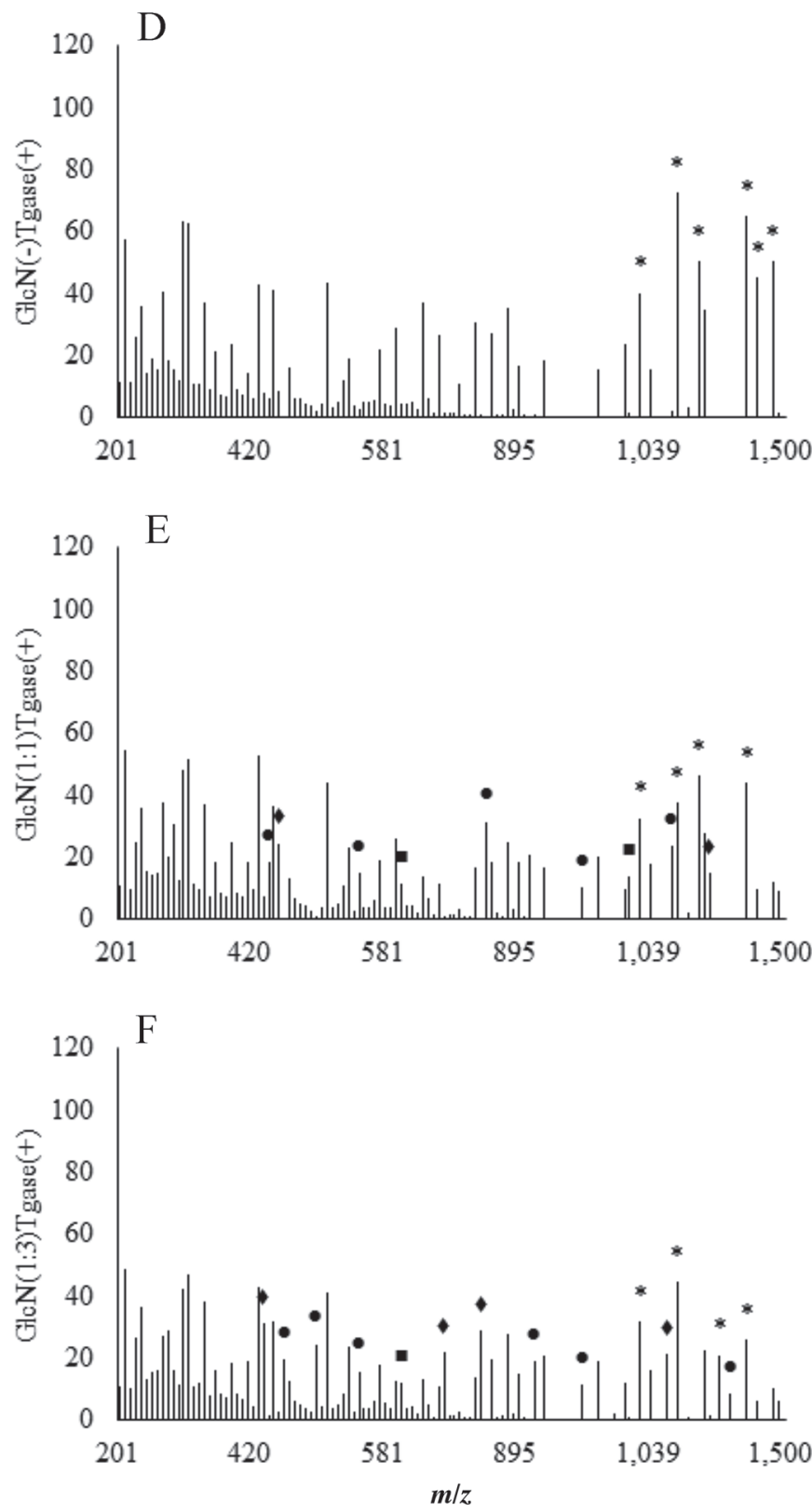

Figure 3. Matrix-assisted laser desorption ionization-time-of-flight mass spectra of whey protein isolate (WPI) conjugated with mixtures of 1:0, 1:1, or 1:3 WPI:glucosamine (GlcN; protein to sugar ratio) incubated with or without enzymatic reaction of transglutaminase (Tgase). Filled squares indicate glycated peptides though release of $1 \mathrm{~mol}$ of $\mathrm{H}_{2} \mathrm{O}$ via non-enzymatic glycation (Maillard reaction). Filled rhombuses indicate glycated peptides through a release of $1 \mathrm{~mol}$ of $\mathrm{H}_{2} \mathrm{O}$ and $1 \mathrm{~mol}$ of $\mathrm{NH}_{3}$ during glycation with GlcN via non-enzymatic glycation (Maillard reaction). Filled circles indicate glycated peptides though a release of $1 \mathrm{~mol}$ of $\mathrm{NH}_{3}$ during glycation with GlcN via enzymatic glycation (transglutaminase). Stars refer to shifts that might represent cross-links induced by transglutaminase. 


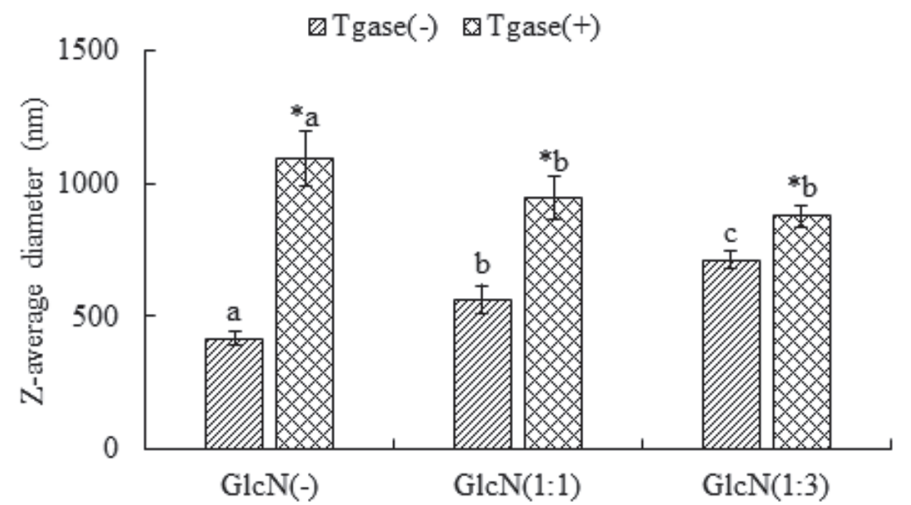

Figure 4. Average particle size of whey protein isolate (WPI) conjugates generated via different combinations of glucosamine (GlcN) and transglutaminase (Tgase). ${ }^{*}$ Significant difference compared with WPI treated without Tgase at the same GlcN level. Different letters for WPI \pm Tgase indicate a significant difference $(P<0.05)$. Error bars indicate $\pm \mathrm{SD}$.

that the hydrophilic sugar residues on the WPI+GlcN conjugates oriented toward water, decreasing the surface hydrophobicity (Hrynets et al., 2013).
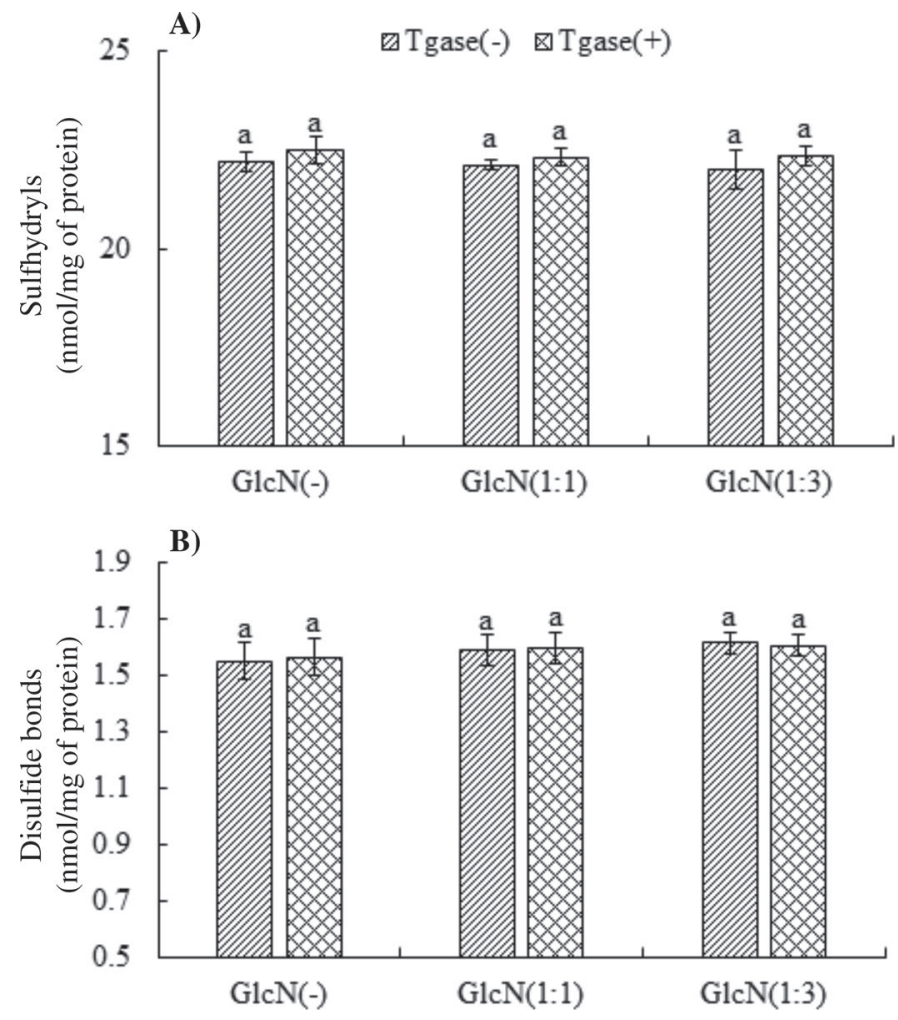

Figure 5. Sulfhydryls and disulfide bonds of whey protein isolate (WPI) conjugates generated via different combinations of glucosamine $(\mathrm{GlcN})$ and transglutaminase (Tgase). Different letters (a) for WPI \pm Tgase indicate a significant difference $(P<0.05)$. Error bars indicate $\pm \mathrm{SD}$.

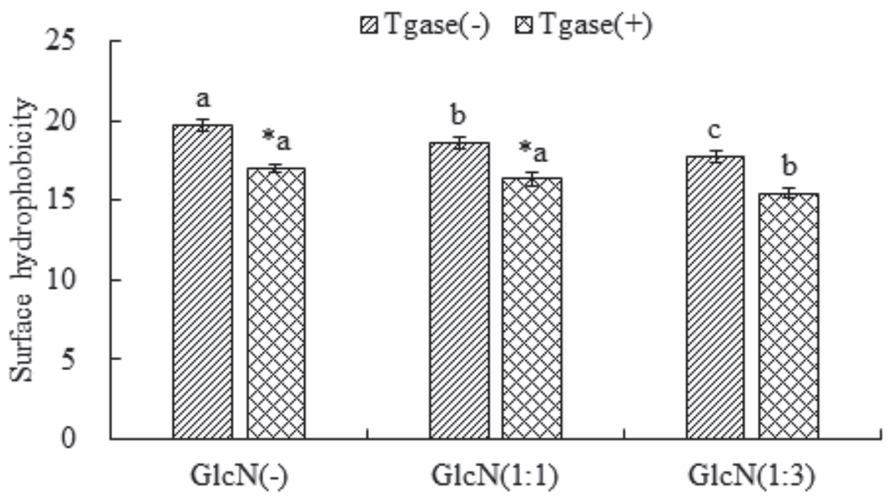

Figure 6. Surface hydrophobicity of whey protein isolate (WPI) conjugates generated via different combinations of glucosamine (GlcN) and transglutaminase (Tgase). ${ }^{*}$ Significant difference compared with WPI treated without Tgase. Different letters $(\mathrm{a}-\mathrm{c})$ for WPI \pm Tgase indicate a significant difference $(P<0.05)$. Error bars indicate $\pm \mathrm{SD}$.

When WPI conjugation was catalyzed by Tgase, protein polymers with higher molecular weights were formed through cross-linking, as shown by DLS and MALDI-TOF-MS analyses. The intramolecular bonds of WPI cross-linked by Tgase may lead to relatively compact polymerized WPI molecules. Compact protein polymers may partly hide their hydrophobic residues inside the aggregates and thus prevent hydrophobic associations. This could explain the significantly lower surface hydrophobicity in WPI treated with Tgase (Figure 6). Others have also reported a decrease in the surface hydrophobicity of whey protein treated with Tgase (Eissa and Khan, 2006). However, some studies have reported that Tgase-induced protein cross-linking resulted in enhanced surface hydrophobicity (Jiang and Zhao, 2010; Song and Zhao, 2014). In these studies, the hydrophobic regions of proteins might be more exposed due to the relatively higher incubation temperature $\left(37^{\circ} \mathrm{C}\right)$.

In the present study, the cross-links between WPI (Figure 1A) were partly inhibited by the reactions between GlcN and WPI (Figure 1B) in the presence of GlcN. Glycation between GlcN and WPI (Figure 1B) not only decreased the degree of polymerization through Tgase-induced cross-links (Figure 1A) but also increased the surface hydrophilicity, because GlcN has good hydrophilicity. As a result, the surface hydrophobicity of the WPI+Tgase samples decreased with the increase of GlcN (Figure 6).

\section{Emulsion Properties of WPI Conjugates}

Among the properties of WPI that are favored for industrial application are the abilities to emulsify fat and to form stable emulsions (Hrynets et al., 2013). 
We determined the emulsifying properties of WPI samples at both low $(0.02 M)$ and high $(0.2 M) \mathrm{NaCl}$ concentrations (Figure 7). At low $\mathrm{NaCl}$ concentrations $(0.02 M)$, both EAI and ESI were significantly higher for the WPI+GlcN conjugates than for the original WPI (Figures 7A and B). Improvement in the emulsifying properties of WPI+GlcN conjugates could change the amphiphilic properties of WPI and more effectively prevent the coalescence of oil droplets (Liu and Zhong, 2012; Hrynets et al., 2013). In a previous study, the emulsifying ability and emulsion stability of an emulsion made with WPI+dextran conjugates were enhanced compared with those of original WPI (Zhu et al., 2010). The WPI treated with Tgase had higher EAI and ESI values than non-enzymatic WPI+GlcN conjugates at each ratio of GlcN $20 \mathrm{mM} \mathrm{NaCl}$ (Figures 7A and B). The Tgase-cross-linked WPI might form a physically strong protective layer around the oil droplets, which would inhibit the coalescence and Ostwald ripening that often occur in oil-in-water emulsion systems. This result was in accordance with previous studies (Ma et al., 2012; Jiang and Xiong, 2015).
In addition, the Tgase-catalyzed conjugation of WPI with GlcN might also promote the emulsifying function of modified WPI. In another study, whey protein concentrate treated with Tgase was remarkably stable, with no phase separation (Clare and Daubert, 2011). It is interesting that the EAI and ESI of WPI+Tgase showed a GlcN dose-dependent decrease (Figures 7A and B). Because Tgase can catalyze cross-links between peptide strands or incorporation of GlcN, the addition of GlcN reduces the cross-links between peptides, as evidenced by MALDI-TOF-MS and DLS, and thus reduces the degree of polymerization catalyzed by Tgase. This lower degree of WPI polymerization may lead to thinner protein coats on the oil droplets, seen as a decrease of EAI and ESI with the increase of GlcN dose in WPI treated with Tgase (Figures 7A and B). Jiang and Xiong (2015) showed that $\beta$-casein polymers, induced by Tgase, had remarkably improved emulsion stability when compared with monomeric $\beta$-casein. Conversely, Jiang and Zhao (2010) found that extensive cross-linking of casein had an adverse effect on emulsion stability. In the present study, treatment of WPI with Tgase at a
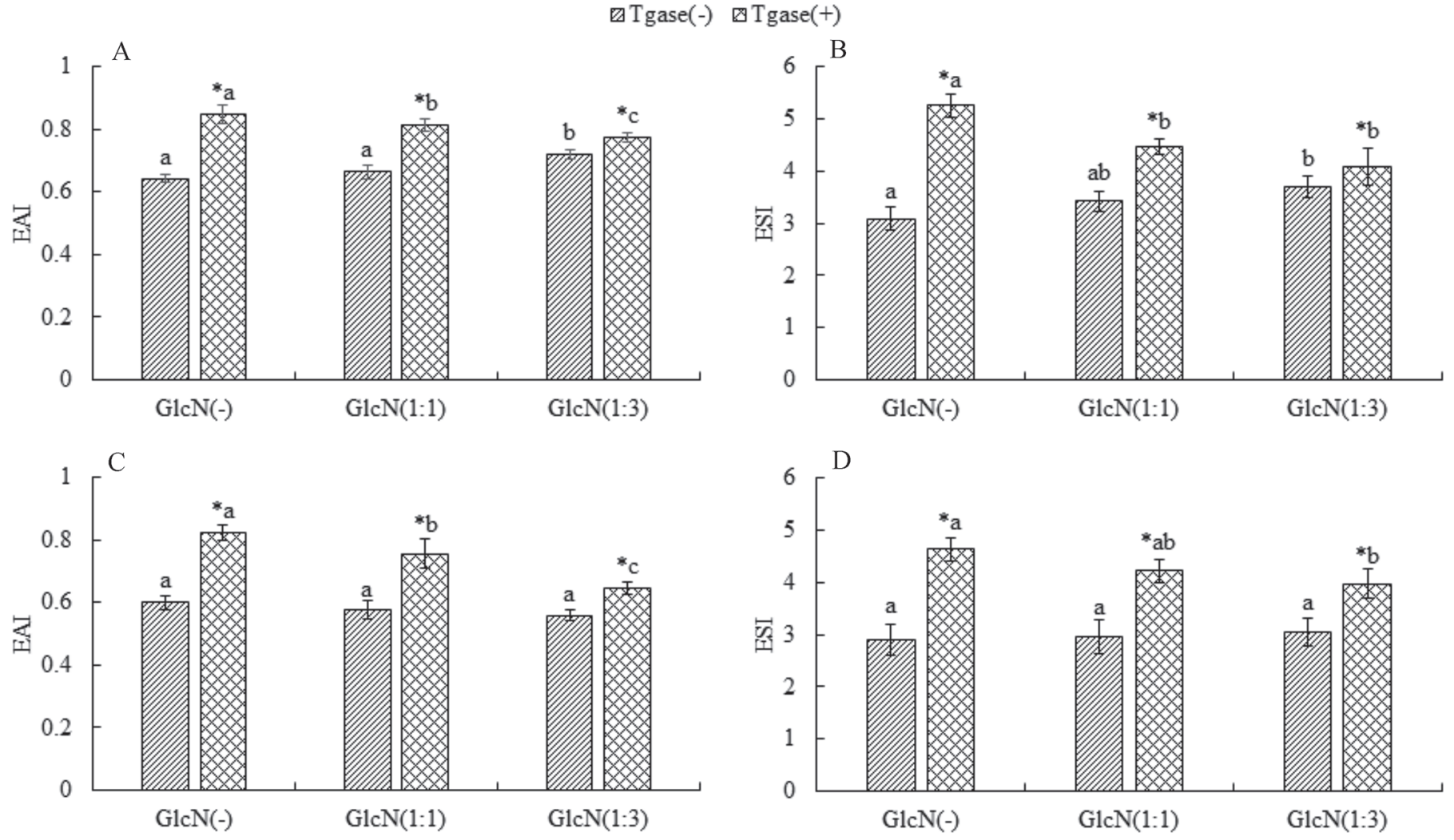

Figure 7. Effect of glucosamine (GlcN) and transglutaminase (Tgase) on the emulsifying activity index (EAI) and emulsion stability index (ESI) of whey protein isolate (WPI) conjugates subjected to emulsification with soybean oil with $0.02 M(\mathrm{~A}$ and $\mathrm{B})$ and $0.2 M(\mathrm{C}$ and $\mathrm{D}) \mathrm{NaCl}$. ${ }^{*}$ Significant difference compared with WPI treated without Tgase. Different letters $(\mathrm{a}-\mathrm{c})$ for WPI \pm Tgase indicate a significant difference $(P$ $<0.05)$. Error bars indicate $\pm \mathrm{SD}$ 
low temperature $\left(25^{\circ} \mathrm{C}\right.$ for $\left.12 \mathrm{~h}\right)$ might lead to moderate cross-linking between proteins, which would adsorb at the interface of the oil droplets, forming an effective steric barrier and conferring improved emulsion stability. It has been reported that cross-linking induced by Tgase could increase the thickness of and enhance the physical stability of the interfacial protein layer (Ma et al., 2012). Another study found that a lower degree of cross-linking of $\beta$-lactoglobulin catalyzed by Tgase improved the stability of the emulsions (Færgemand et al., 1998).

At high $\mathrm{NaCl}$ concentrations $(200 \mathrm{mM})$, the EAI and ESI values of WPI+GlcN conjugates treated without Tgase did not significantly change with increasing GlcN in emulsions (Figures $7 \mathrm{C}$ and $\mathrm{D}$ ). The negative charges present on the surface of WPI+GlcN conjugates could have been neutralized by the positive sodium ions at higher $\mathrm{NaCl}$ concentrations (Wu et al., 2015), and this might have masked the effect of glycation on the emulsifying properties of the WPI+GlcN conjugates. One study found that 50 to $100 \mathrm{mM} \mathrm{NaCl}$ effectively screened negative charges on whey proteins (Ryan et al., 2012). However, the external ionic charges may have had slight effects on the EAI and ESI of WPI cross-linked by Tgase. As a result, the EAI and ESI of the high salt emulsion stabilized with WPI+Tgase showed changes due to steric hindrance that were similar to the low salt $(0.02 \mathrm{M})$ emulsion (Figure 7).

The CLSM images clearly showed the protein coat on the outside of the oil droplets on the oil-water interface (indicated by the red arrow; Figures 8 and 9). For emulsions that were prepared with WPI+GlcN conjugates, the dispersion of oil droplets increased with the increase of GlcN dose (Figure 8), confirming that WPI+GlcN

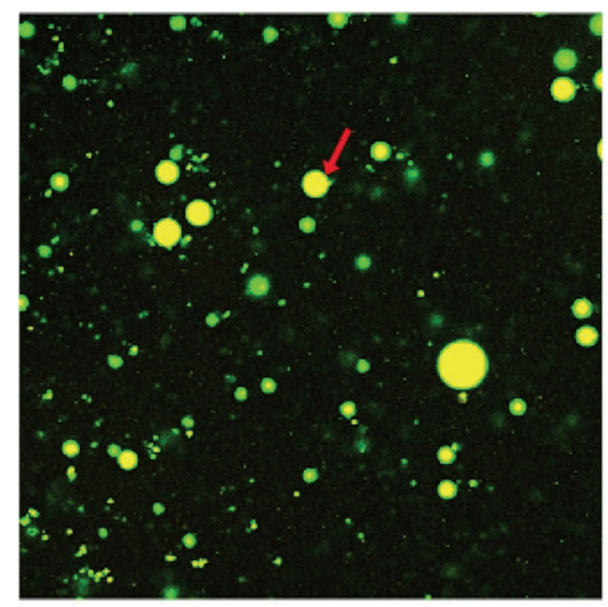

A

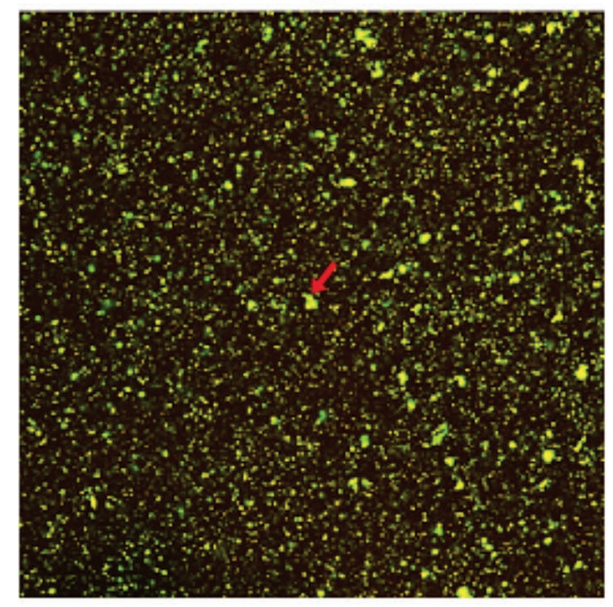

D

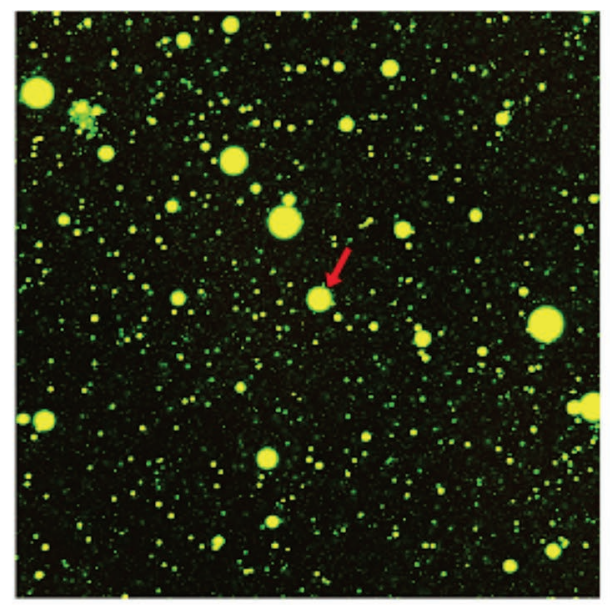

B

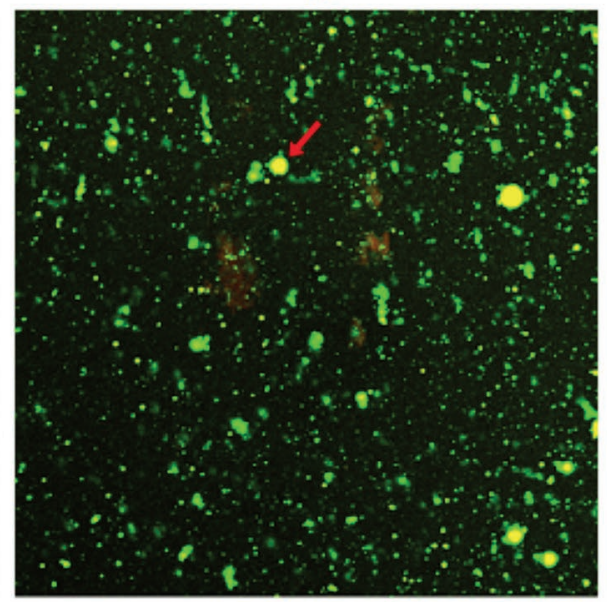

E

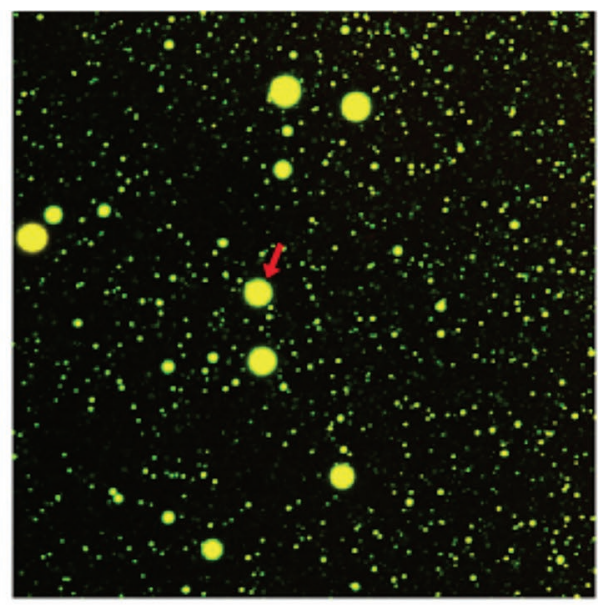

C

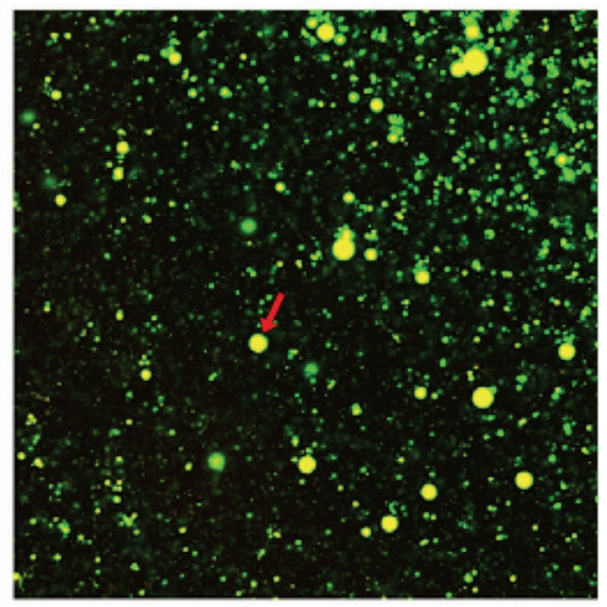

F

Figure 8. Photomicrographs of oil emulsions stabilized with different whey protein isolate (WPI) conjugates at a lower NaCl concentration (20 mM). (A) GlcN-, Tgase-; (B) GlcN 1:1, Tgase-; (C) GlcN 1:3, Tgase-; (D) GlcN-, Tgase+; (E) GlcN 1:1, Tgase+; (F) GlcN 1:3, Tgase+. GlcN = glucosamine; Tgase $=$ transglutaminase. Protein on the oil-water interface is indicated by an arrow (red). Bright (yellow) spots indicate oil droplets; less intense (green) spots indicate protein. Color version available online. 
conjugates prevented coalescence of oil droplets. The emulsions prepared with Tgase-treated WPI showed smaller oil-droplet sizes at each GlcN ratio compared with the WPI incubated with GlcN only (Figure 8), supporting the idea that Tgase-cross-linked WPI might form a physically stronger protective layer around the oil droplets. In addition, the CLSM images of the emulsions supported the aforementioned changes in EAI and ESI values at $200 \mathrm{~m} M \mathrm{NaCl}$ (Figure 9).

For a clearer understanding of the influence of Tgase and GlcN on the emulsifying activity and emulsionstabilizing ability of WPI, we have provided a proposed schematic of the Tgase-induced cross-linking and GlcN conjugation in Figure 10. Without Tgase, WPI+GlcN conjugates were formed by non-enzymatic glycation (Maillard reaction). The incorporation of GlcN into the protein changed the amphiphilic properties of WPI, improving their emulsifying activity and emulsion-stabilizing ability compared with WPI that were treated without GlcN under lower $\mathrm{NaCl}$ concentrations (20 $\mathrm{m} M)$. However, the emulsifying activity and emulsionstabilizing ability of WPI did not change with increase of GlcN under higher $\mathrm{NaCl}$ concentration $(200 \mathrm{mM}$ ). The addition of Tgase catalyzed moderate cross-linking between proteins. Cross-linked WPI formed polymers of larger size, forming a thicker steric barrier on oils due to enhanced protein-protein interaction and improving the physical stability of the emulsions. However, covalent linkages between GlcN and WPI formed via both nonenzymatic (Maillard reaction) and enzymatic (Tgase) glycation may partly inhibit the degree of cross-linking of the WPI; as a result, the size of the WPI polymers become smaller with the increase in GlcN dose. The smaller WPI polymers might lead to a thinner protein

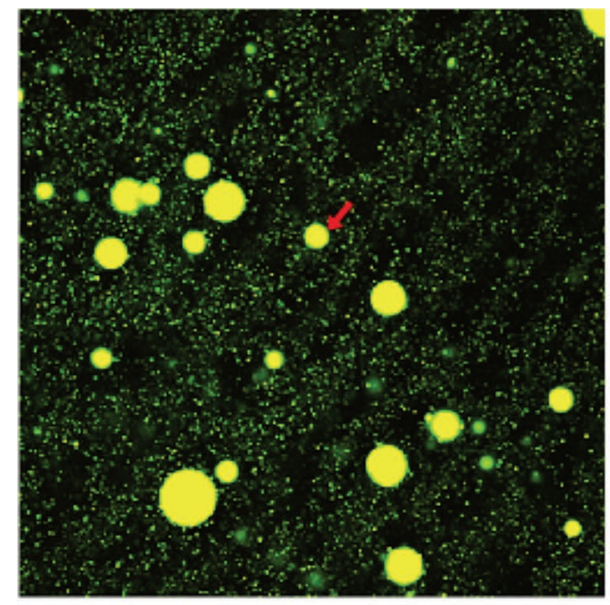

A

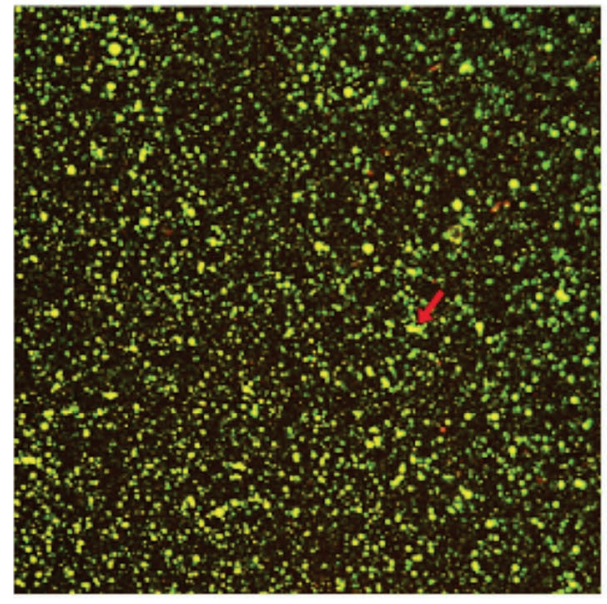

D

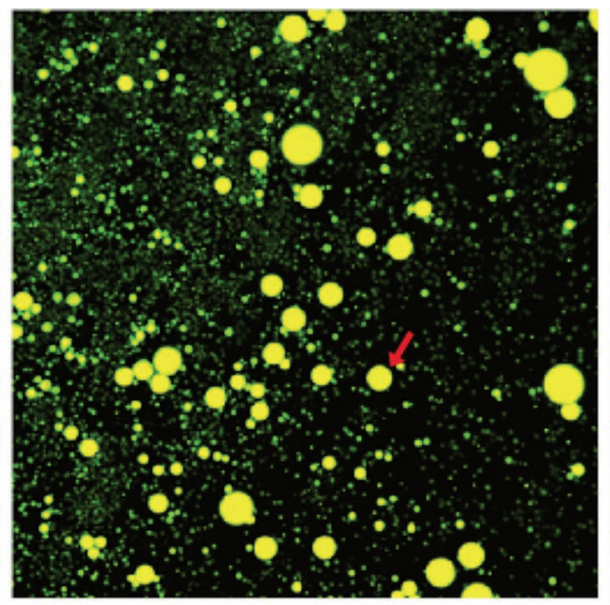

B

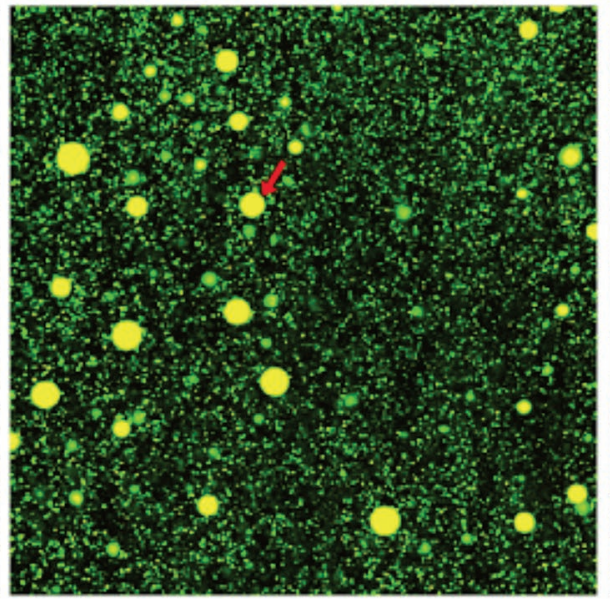

E

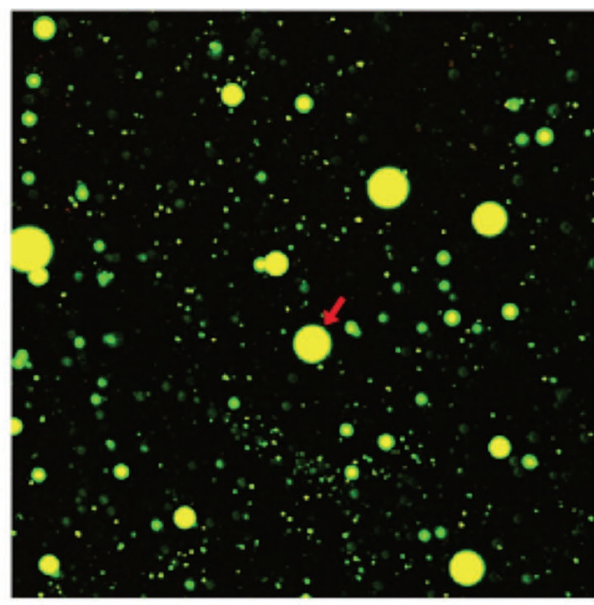

$\mathrm{C}$

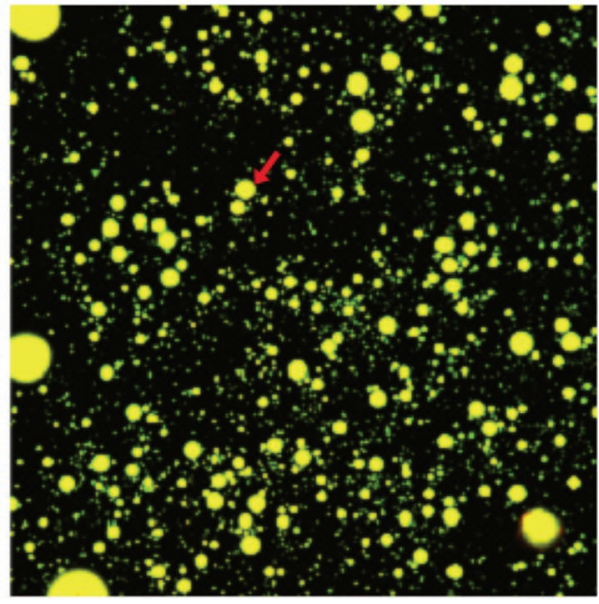

$\mathrm{F}$

Figure 9. Photomicrographs of oil emulsions stabilized with different whey protein isolate (WPI) conjugates at a higher NaCl concentration (0.2 M) . (A) GlcN-, Tgase-; (B) GlcN 1:1, Tgase-; (C) GlcN 1:3, Tgase-; (D) GlcN-, Tgase+; (E) GlcN 1:1, Tgase+; (F) GlcN 1:3, Tgase+. GlcN = glucosamine; Tgase $=$ transglutaminase. Protein on the oil-water interface is indicated by an arrow (red). Bright (yellow) spots indicate oil droplets; less intense (green) spots indicate protein. Color version available online. 


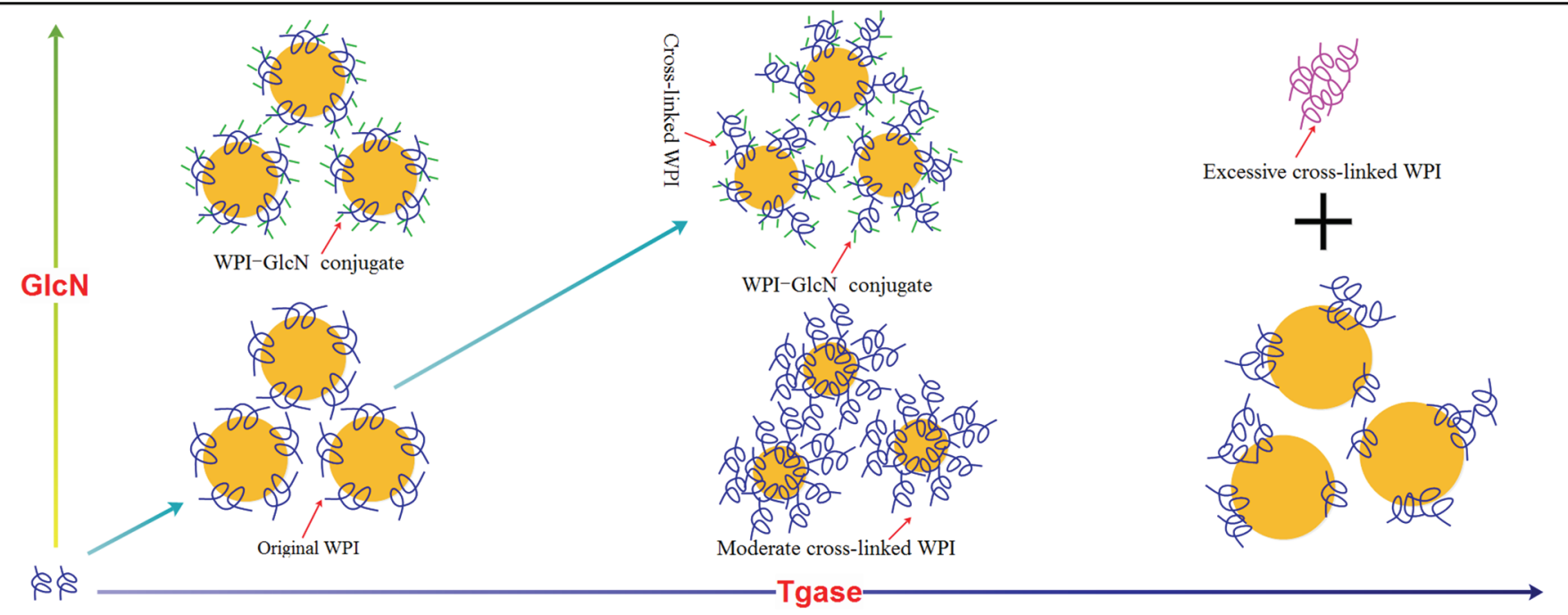

Figure 10. Schematic of an adverse effect of incorporated glucosamine (GlcN) residue on emulsifying properties of transglutaminase (Tgase)cross-linked whey protein isolate (WPI). The WPI is the squiggly line (blue); oil globules are the circular spots (brown). Color version available online.

layer adsorbed on the oil-water interface, and lower stability of the emulsions.

\section{CONCLUSIONS}

Addition of either GlcN or Tgase can improve the emulsifying activity and emulsion-stabilizing ability of WPI. In the absence of Tgase, GlcN can be incorporated into WPI by non-enzymatic glycation (Maillard reaction) between the carbonyl group of a reducing sugar and the free amino groups in the protein. The WPI conjugates adsorb at the oil-water interface to form a protective layer that prevents oil droplets from coalescing as a result of hydrophilic GlcN residues orienting toward water. The Tgase-catalyzed cross-links between WPI molecules that lead to moderate polymerization between WPI molecules seemed ideal. Larger-sized polymers create a thicker and physically stronger steric barrier around the droplets, effectively reducing coalescence and Ostwald ripening in the oil-in-water emulsion. Our work demonstrated the interesting discovery that the addition of GlcN can decrease the emulsifying activity and emulsion stability of WPI cross-linked by Tgase. Concentration of $\mathrm{NaCl}$ had a strong influence on the emulsifying activity and emulsion stability of WPI+GlcN conjugates, limiting their potential use. On the other hand, the improved emulsifying activity and emulsion stability with WPI cross-linked by Tgase were negligibly affected by $\mathrm{NaCl}$ concentration. This finding shows that Tgase has a high potential for use as an emulsifying stabilizer in food products that contain a relatively high $\mathrm{NaCl}$ concentrations.

\section{ACKNOWLEDGMENTS}

This work was supported by the National Natural Science Fund for Young Scholars (grant numbers 31601497, 31401515), the China Postdoctoral Science Foundation Project (grant number 2016M591857), the Shaanxi Province Science and Technology Research and Development Project (grant number 2015NY022), the National Center of Meat Quality and Safety Control (Nanjing Agricultural University; grant numbers M2015K06; M2016K01), the Chinese Universities Scientific Fund (grant number 2452015062) and the Earmarked Fund for Modern Agro-industry Technology Research System, China (grant number nycytx42-G5-01). All authors read, commented on, and approved the final manuscript.

\section{REFERENCES}

Arbia, W., L. Arbia, L. Adour, and A. Amrane. 2013. Chitin extraction from crustacean shells using biological methods-A review. Food Technol. Biotechnol. 51:12-25.

Clare, D. A., and C. R. Daubert. 2011. Expanded functionality of modified whey protein dispersions after transglutaminase catalysis. J. Food Sci. 76:C576-C584.

Eissa, A. S., and S. A. Khan. 2006. Modulation of hydrophobic interactions in denatured whey proteins by transglutaminase enzyme. Food Hydrocoll. 20:543-547. 
Færgemand, M., J. Otte, and K. B. Qvist. 1998. Emulsifying properties of milk proteins cross-linked with microbial transglutaminase. Int. Dairy J. 8:715-723.

Feng, X., C. Li, N. Ullah, J. Cao, Y. Lan, W. Ge, R. M. Hackman, Z. $\mathrm{Li}$, and L. Chen. 2015a. Susceptibility of whey protein isolate to oxidation and changes in physicochemical, structural, and digestibility characteristics. J. Dairy Sci. 98:7602-7613.

Feng, X., C. Li, N. Ullah, R. M. Hackman, L. Chen, and G. Zhou. 2015b. Potential biomarker of myofibrillar protein oxidation in raw and cooked ham: 3-nitrotyrosine formed by nitrosation. J. Agric. Food Chem. 65:10957-10964. https://doi.org/10.1021/acs. jafc.5b04107.

Hrynets, Y., M. Betti, and M. Ndagijimana. 2014. Transglutaminasecatalyzed glycosylation of natural actomyosin (NAM) using glucosamine as amine donor: Functionality and gel microstructure. Food Hydrocoll. 36:26-36.

Hrynets, Y., M. Ndagijimana, and M. Betti. 2013. Non-enzymatic glycation of natural actomyosin (NAM) with glucosamine in a liquid system at moderate temperatures. Food Chem. 139:1062-1072.

Jiang, J., and Y. L. Xiong. 2015. Role of interfacial protein membrane in oxidative stability of vegetable oil substitution emulsions applicable to nutritionally modified sausage. Meat Sci.109:56-65.

Jiang, S. J., and X. H. Zhao. 2010. Transglutaminase-induced crosslinking and glucosamine conjugation in soybean protein isolates and its impacts on some functional properties of the products. Eur. Food Res. Technol. 231:679-689.
Liu, G., and Q. Zhong. 2012. Glycation of whey protein to provide steric hindrance against thermal aggregation. J. Agric. Food Chem. 60:9754-9762.

Ma, H., P. Forssell, P. Kylli, A.-M. Lampi, J. Buchert, H. Boer, and R. Partanen. 2012. Transglutaminase catalyzed cross-linking of sodium caseinate improves oxidative stability of flaxseed oil emulsion. J. Agric. Food Chem. 60:6223-6229.

Ryan, K. N., B. Vardhanabhuti, D. P. Jaramillo, J. H. V. Zanten, J. N. Coupland, and E. A. Foegeding. 2012. Stability and mechanism of whey protein soluble aggregates thermally treated with salts. Food Hydrocoll. 27:411-420.

Sarbon, N. M., F. Badii, and N. K. Howell. 2015. The effect of chicken skin gelatin and whey protein interactions on rheological and thermal properties. Food Hydrocoll. 45:83-92.

Song, C.-L., and X.-H. Zhao. 2014. Structure and property modification of an oligochitosan-glycosylated and crosslinked soybean protein generated by microbial transglutaminase. Food Chem. 163:114-119.

Wu, J., M. Shi, W. Li, L. Zhao, Z. Wang, X. Yan, W. Norde, and Y. Li. 2015. Pickering emulsions stabilized by whey protein nanoparticles prepared by thermal cross-linking. Colloids Surf. B Biointerfaces 127:96-104.

Zhu, D., S. Damodaran, and J. A. Lucey. 2010. Physicochemical and emulsifying properties of whey protein isolate (WPI) - dextran conjugates produced in aqueous solution. J. Agric. Food Chem. 58:2988-2994. 